Document downloaded from:

http://hdl.handle.net/10251/180570

This paper must be cited as:

Cirugeda, E.; Plancha, E.; Hidalgo, V.; Calero, S.; Rieta, JJ.; Alcaraz, R. (2019). Predicting Electrical Cardioversion Outcome in Persistent Atrial Fibrillation Through Multiscale Entropy Analysis. IEEE. 1-4. https://doi.org/10.1109/EHB47216.2019.8969889

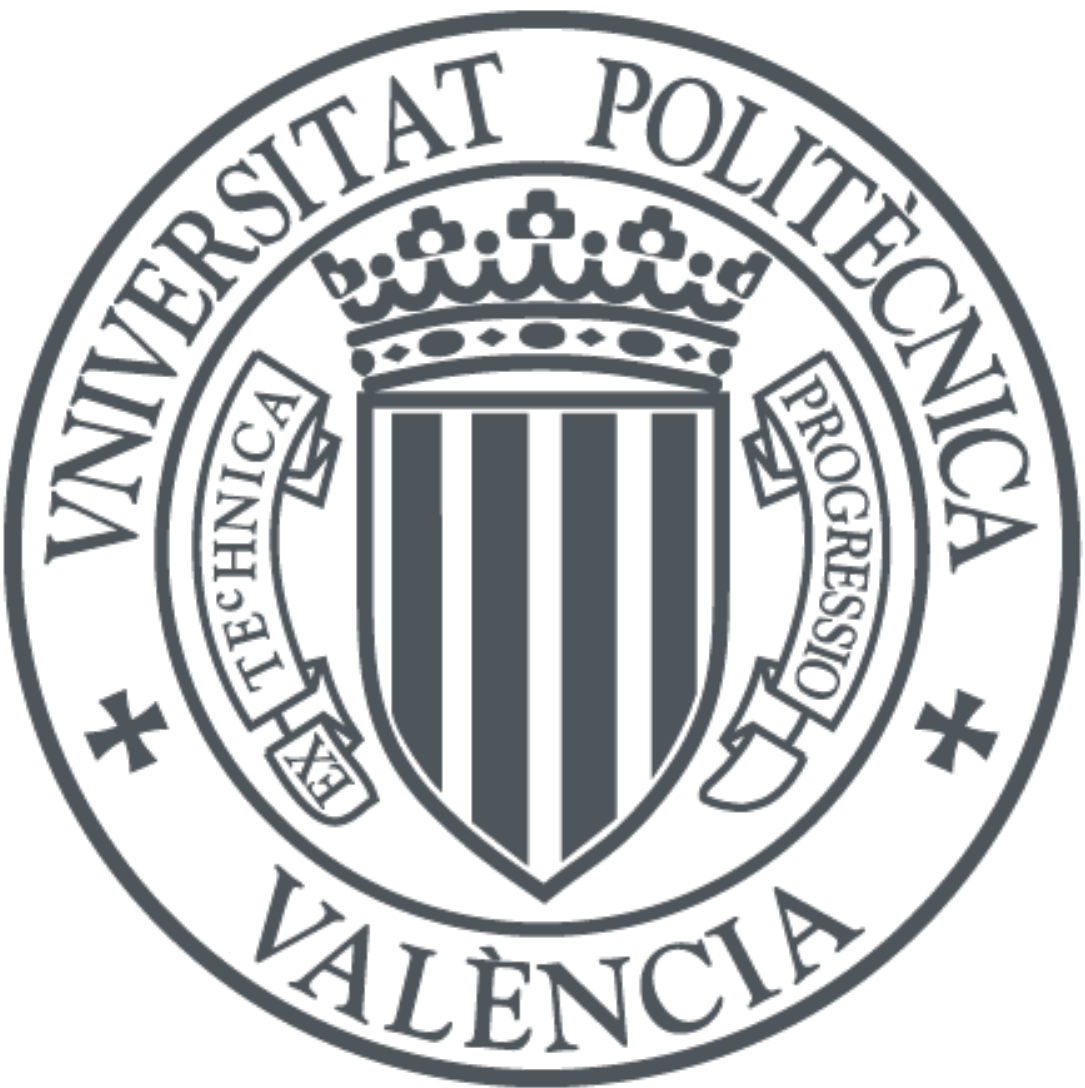

The final publication is available at

https://doi.org/10.1109/EHB47216.2019.8969889

Copyright IEEE

Additional Information 


\title{
Predicting Electrical Cardioversion Outcome in Persistent Atrial Fibrillation Through Multiscale Entropy Analysis
}

\author{
E. Cirugeda ${ }^{1}$, E. Plancha ${ }^{2}$, V.M. Hidalgo ${ }^{3}$, S. Calero ${ }^{3}$, J.J. Rieta ${ }^{4}$, R. Alcaraz ${ }^{1}$ \\ ${ }^{1}$ Research Group in Electronic, Biomedical and Telecommunication Engineering, \\ University of Castilla-La Mancha,Cuenca, Spain, \{eva.cirugeda, raul.alcaraz\}@uclm.es \\ ${ }^{2}$ FISABIO - Department of Internal Medicine, Hospital Francesc de Borja, Gandía, Spain, plancha_eva@gva.es \\ ${ }^{3}$ Cardiac Arrhythmia Department, University Hospital of Albacete, Albacete, Spain, \\ \{sofia.caleron,vmhidalgoolivares\}@gmail.com \\ ${ }^{4}$ BioMIT.org, Electronic Engineering Depart., Universitat Politecnica de Valencia, Valencia, Spain,
}

\begin{abstract}
Atrial Fibrillation (AF) is the most commonly sustained cardiac arrhythmia and the major cause of cardiovascular morbidity and mortality. Because of its wide availability and initial effectiveness, electrical cardioversion (ECV) is the primary method used for reverting this arrhythmia to normal sinus rhythm (NSR). However, this procedure presents some collateral effects, and barely $80 \%$ of the patients prevail in NSR after 1 month. Thus, being able to predict the outcome of ECV before its application is of great interest in clinical practice, so cardiac complications in patients with high probability of early AF recurrence could be prevented. For that purpose, this work characterizes atrial activity (AA) in patients with persistent AF, before ECV, by means of nonlinear multiscale dynamics, particularly composite multiscale entropy (CMSE), and compares its performance with other recently used parameters, such as, dominant atrial frequency, AA's amplitude and sample entropy. The results show that characterizing AA by means of CMSE predicts ECV outcome with an accuracy above $90 \%$, whereas the remaining parameters only forecast correctly about $70 \%$ of the analyzed patients. As a conclusion, using complexity techniques at different time scales for AA characterization increases the probability of correctly predicting AF recurrence.
\end{abstract}

Keywords- Atrial Fibrillation, Electrical Cardioversion, Composite Multiscale Entropy, Sample Entropy (SampEn), Atrial Activity

\section{INTRODUCTION}

Atrial fibrillation (AF) is the most commonly sustained cardiac arrhythmia, affecting 33 million individuals worldwide. It is defined as a tachyarrhythmia characterized by extremely rapid and uncoordinated atrial activations with consequent deterioration of the mechanical atrial function [1]. In fact, this arrhythmia is observed and diagnosed in the ECG by the fact that $P$ waves are replaced by fibrillatory waves ( $f$ waves), which present variable size, amplitude and timing. Additionally, although the QRS complex morphology remains unchanged, during AF the ventricular response is often rapid (90-170 beats per minute) and highly irregular [1-3]
Attending to its progressive nature, AF is classified into four major groups: paroxysmal, persistent, long-standing persistent, and permanent [1]. Although AF is initially nonsustained, it promotes electrical and mechanical changes in the atrial, thus contributing to maintain the arrhythmia [1]. In this stage, persistent $\mathrm{AF}$ is a relevant risk factor of stroke and mortality, and its treatment is mainly focused on restoring normal sinus rhythm (NSR). Indeed, electrical cardioversion (ECV) is the primary used method for that purpose [2]. This procedure consists on delivering one or more controlled transthoracic electrical shocks to the patient until NSR is restored or the maximum allowed power is applied to the heart [3]. To increase the probability of NSR maintenance, patients are often treated with antiarrhythmic drugs before ECV. Nonetheless, nearly $20 \%$ of the patients still relapse to AF within 30 days, $40-60 \%$ revert to AF within 3 months and around $60-80 \%$ within a year [4]. Moreover, ECV is also considered an important cause of collateral effects, such as malignant ventricular arrhythmias, arterial thromboembolism and other related complications [4].

Within this context, being able to characterize and predict ECV outcome before its application is of great clinical interest. In this way, ECV could be avoided in patients with a limited probability of NSR maintenance, thus preventing risks and complications, as well as saving costs for healthy systems. So far, numerous studies have attempted to find either invasive [57] and non-invasive [8-9] predictors of the ECV outcome, but only a few of them have dealt with complexity analysis of the atrial activity (AA) signal [11-14].

The present work aims to continue the proposal in [13], where sample entropy (SampEn) was used to characterize the AA signal and then predict ECV outcome. It deals with the hypothesis that time series derived from complex systems show dynamical variations and structures on different time scales, which SampEn does not account for [15]. Composite multiscale entropy (CMSE) [16] is then considered here in order to improve ECV outcome prediction. Instead of common multiscale entropy, this tool is used due to its improved ability 
TABLE I.

CLINICAL CHARACTERISTICS OF THE ANALIZED PATIENTS

\begin{tabular}{|c|c|c|}
\hline Parameter & NSR Maintenance & AF relapsing \\
\hline Patients & 14 & 21 \\
\hline Men & 4 & 8 \\
\hline Underlying heart disease & 2 & 5 \\
\hline Sustained AF duration (months) & $9.5(1-36)$ & $10.5(1-54)$ \\
\hline Left atrial diameter $(\mathrm{mm})$ & $4.88 \pm 8.26$ & $46.23 \pm 6.20$ \\
\hline
\end{tabular}

to reduce the variability that multiscale entropy presents at large time scales [17].

\section{DATABASE AND METHODS}

\section{A. Database}

The analyzed database consisted of 35 12-lead ECG recordings, sampled at $1024 \mathrm{~Hz}$ and classified as a function of the patients' rhythm one month after ECV: NSR maintenance or AF recurrence. This work only considered ECG recordings from patients who reverted to NSR after only one shock with power of 200J. Table 1 provides clinical characteristics of these patients.

Although the 12 standard leads were available for all ECG recordings, only lead V1 was considered for AA characterization, because it presents the largest $f$ waves [12]. Similarly, only a 1.5 minute-length segment before the electrical shock was analyzed in this work. A more detailed description of this database can be found in [14], where it was also studied.

\section{B. Atrial Activity Characterization}

Before extracting and characterizing the AA signal, the raw ECG was preprocessed. This consisted on removing baseline wandering, power line interference, and high frequency noise. Baseline was estimated by means of a $3^{\text {rd }}$ order Butterworth low-pass filter with a cut-off frequency of $0.8 \mathrm{~Hz}$ and then subtracted. Powerline interference was removed through a notch filter centered on $50 \mathrm{~Hz}$ and $4 \mathrm{~Hz}$ bandwidth. The remaining signal was low-pass filtered at $40 \mathrm{~Hz}$ for highfrequency noise removal [18]. Next, to extract the AA signal, a well-known algorithm based on average QRS complex subtraction was used [19]. Briefly, QRS complexes were detected using a previously published algorithm [20], and QRST length was determined experimentally and set to a $65 \%$ of the mean RR interval. Whether the ECG presented ectopic beats, a two-step cancellation approach was performed. First, a template was computed over the ectopic QRST complexes and they were subsequently cancelled. Secondly, this templatebased cancellation algorithm was applied over the remaining normal QRST complexes. Note that ectopic beats were detected and classified by means of a pattern recognition and thresholding algorithm [21].

For AA characterization, different parameters were considered: AA absolute and normalized amplitudes (i.e., FWA and FWAn), the dominant atrial frequency (DAF) and two complexity measures (i.e., SampEn and CMSE). Considering that the AA signal, referred to as $a a(n)$, is a discrete signal with $N$ samples in length, FWA and FWAn were estimated according to:

$$
\begin{gathered}
F W A=\sqrt{\frac{1}{N} \sum_{i=1}^{N}|a a(i)|^{2}} \\
F W A n=\frac{F W A}{\sqrt{\frac{1}{N_{b}} \sum_{k=1}^{N_{b}}|x(k)|^{2}}}
\end{gathered}
$$

where $x(n)$ denotes the ECG, $k$ indexes the QRS complex and $N_{b}$ is the total number of beats in the ECG signal. The DAF was estimated as the maximum peak between 3 and $12 \mathrm{~Hz}$ over the AA power spectral density (PSD), i.e.,

$$
D A F=\arg \{\max \{P S D(f)\}\}, \quad f \in[3,12] H z
$$

where the power spectral density was computed from $20 \mathrm{~s}$ length windows with $75 \%$ overlapping.

Next, both SampEn and CMSE were estimated from the main atrial wave (MAW) of the AA signal, such as recommended by previous works [11-13]. The MAW was obtained by band-pass filtering the AA with a $4 \mathrm{~Hz}$ bandwidth type II Chebychev filter centered on the DAF. These measures evaluate the self-similarity within a nonstationary time series, so that higher values indicate a less organized behavior. More precisely, SampEn estimates the likelihood ratio that the number of epochs of length $m$ prevail when these epochs are increased in one unit within a tolerance $r$ [15]. From a mathematical point of view, if the MAW is considered as a discrete time series, referred to as $u(n)$ for $n=0: N-1$, the first step to compute SampEn is to form the subvectors $v(i)$ of length $m$. Next, the distance between every pair of vectors $v(i)$ and $v(j)$ is computed and the likelihood of similar patterns of length $m$ and tolerance $r, A^{m}(r)$, is estimated as:

$$
\begin{array}{r}
v(i)=[u(i), u(i+1), \ldots, u(i+-m-1)], \quad i=1: N-m \\
d_{i j}=\max _{k}\{\|v(i+k-1)-v(j+k-1)\|\}, \quad k=1: m, i \neq j \\
A^{m}(r)=\frac{1}{N-m} \sum_{i=1}^{N-m}\left(\frac{1}{N-m-1} \sum_{\substack{i=1 \\
i \neq j}}^{N-m}\left(d_{i j}(r) \leq r\right)\right)
\end{array}
$$

Finally, SampEn is obtained as:

$$
\operatorname{SampEn}(x, m, r, N)=-\log \left\{A^{m+1}(r) / A^{m}(r)\right\}
$$

Unfortunately, this entropy-based index does not account for dynamical variations on different time scales [15]; thus, multiscale entropy has been proposed to overcome this limitation [17]. However, traditional multiscale entropy has shown an increasing variance in the estimated entropy at large time scales due to length restrictions, and several alternatives have been recently proposed, including CMSE [16]. This metric requires computation of a coarse-grained series at each time scale $\tau$, which was here computed from the MAW signal, such as:

$$
y_{j}^{\tau}(k)=\frac{1}{\tau} \sum_{i=(k-1) \tau+j+1}^{k \tau+j-1} u(i), \quad \begin{gathered}
k=1:\lfloor N / \tau\rfloor \\
j=1: \tau
\end{gathered}
$$


Then, CMSE was estimated as the mean of the SampEn values computed from the coarse-grained series for all considered time scales, i.e.:

$$
\operatorname{CMSE}(m, r, N, \tau)=\frac{1}{\tau} \sum_{j=1}^{\tau} \operatorname{SampEn}\left(y_{j}^{\tau}, m, r, N\right)
$$

The MAW signal was divided in 30 s intervals, thus ensuring a robust enough estimation by preserving a minimum segment length of 1500 samples at the maximum considered scale $\tau=20$. SampEn parameters were set to $r=0.2$ times the standard deviation of the signal and $m=2$ [15].

\section{Statistical Analysis}

A Kolmogorov-Smirnov test was used to assess normality of data. For indices meeting parametric test assumptions, statistical differences between patients maintaining NSR and relapsing to AF were estimated by means of a $t$-Student's test, whereas Kruskal-Wallis test was employed for non-normal features. On the other hand, a receiver-operating characteristic curve (ROC) was considered to evaluate classification performance of each metric. The area under the ROC curve (AUC) was taken as an approximation of the diagnostic accuracy reached by each index.

\section{RESULTS}

Figure 1 shows a comparative between median values of CMSE for NSR maintenance and AF relapsing groups at different time scales $\tau$. A similar behavior is found between groups, although AF relapsing group shows slightly higher entropy values. Statistical tests were performed for every time scale and the Kolmogorov-Smirnov test revealed not normal distributions for most of them. Thus, the Kruskal-Wallis test provided statistical significance $(p<0.05)$ at all scales, but $\tau=$ $\{3,4,5\}$. Additionally, the AUC remained always above $68.2 \%$. As a summary, Table 2 provides median values, statistical significance $(p)$, and AUC for some of the considered time scales $\tau$. Nonetheless, it should be noted that the maximum AUC of $90.2 \%$ was achieved at $\tau=19$.

Regarding the remaining parameters computed from the AA signal, Table 3 presents median values, statistical significance $(p)$, and AUC for both, patients being able to maintain NSR and patients that relapsed to AF after one month. Moreover, Figure 2 shows their graphical distribution in boxplots. As it can be observed, patients unable to maintain NSR exhibits generally, higher values of the three indices DAF, SampEn and

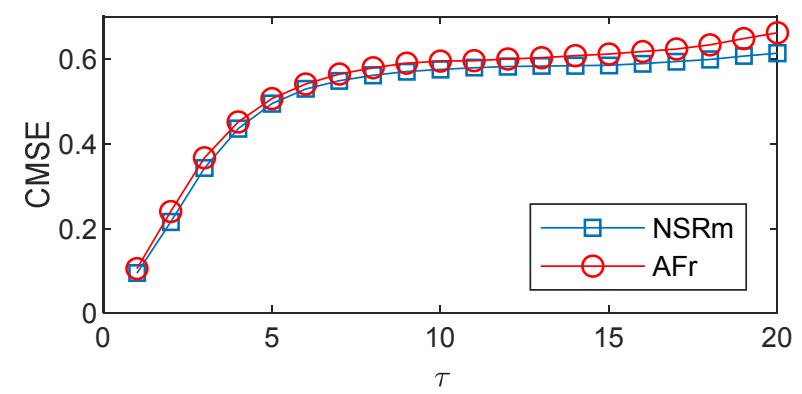

Fig. 1. CMSE median values for different scales $(\tau)$. In blue NSR maintainance (NSRm) and in red AF recurrence (AFr).
TABLE 2. CMSE VALUES OBTAINED AT DIFFERENT TIME SCALES. MEDIAN VALUES, INTERQUARTILIC RANGES, STATISTICAL SIGNIFICANCE AND AUC ARE PRESENTED

\begin{tabular}{|l|l|l|l|l|}
\hline $\boldsymbol{\tau}$ & NSR Maintenance & AF recurrence & $\boldsymbol{p}$ & AUC (\%) \\
\hline 1 & $0.095(0.064)$ & $0.106(0.021)$ & 0.033 & 71.1 \\
\hline 5 & $0.494(0.271)$ & $0.506(0.050)$ & 0.063 & 68.5 \\
\hline 10 & $0.575(0.170)$ & $0.595(0.020)$ & 0.013 & 74.7 \\
\hline 15 & $0.585(0.108)$ & $0.612(0.041)$ & 0.001 & 86.3 \\
\hline 20 & $0.613(0.093)$ & $0.622(0.109)$ & 0.001 & 89.6 \\
\hline
\end{tabular}

TABLE 3. CLASSIFICTION PERFORMANCE OF THE ANALYZED PARAMETERS. MEDIAN VALUES, INTERQUARTILIC RANGES, STATISTICAL SIGNIFICANCE AND AUC ARE PRESENTED

\begin{tabular}{|l|l|l|l|l|}
\hline Parameter & NSR Maintenance & AF recurrence & $\boldsymbol{p}$ & AUC (\%) \\
\hline FWAn & $0.095(0.055)$ & $0.106(0.064)$ & 0.008 & 71.2 \\
\hline DAF & $5.125(1.250)$ & $5.625(1.500)$ & 0.075 & 69.7 \\
\hline SampEn & $0.095(0.064)$ & $0.106(0.021)$ & 0.033 & 71.1 \\
\hline CMSE 19 & $0.607(0.094)$ & $0.647(0.094)$ & 0.001 & 90.2 \\
\hline
\end{tabular}

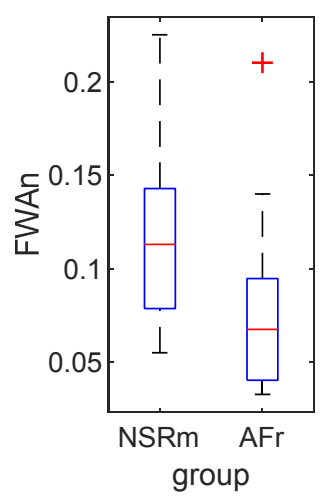

(a)

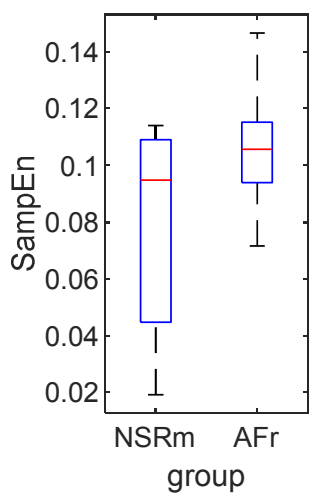

(c)

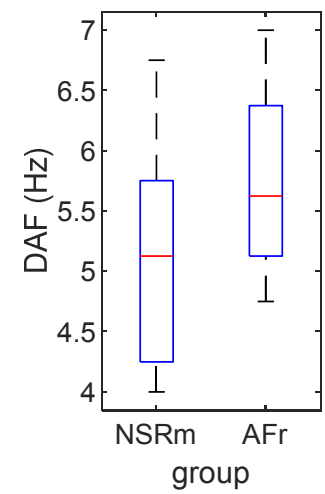

(b)

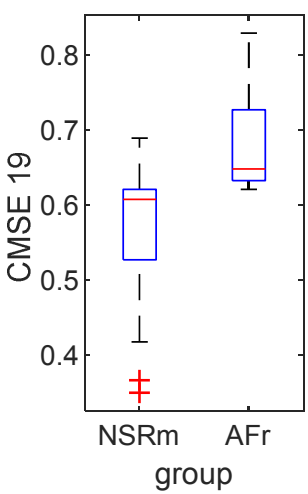

(d)
Fig. 2. Graphical distribution of the values obtained for the analyzed indices: (a) FWAn, (b) DAF, (c) SampEn, and (d) CMSE (for time-scale $\tau=19$ ) for both groups, NSR maintenance (NSRm) and AF recurrence (AFr).

CMSE than NSR maintenance. Note that the optimal scale is only given for CMSE, and FWA values are not presented as they showed worst performance than FWAn.

\section{DISCUSION AND CONCLUSIONS}

The DAF, SampEn and CMSE indexes provide higher values for patients who relapsed to AF, while the FWAn does not. These results are in line with other previous works that also associate lower values with a higher AA organization and, 
consequently, higher probability of NSR maintenance one month after ECV [11-13]. The DAF and FWAn have been widely established in the literature as the best predictors of ECV outcome [13]. However, the obtained results show how complexity measures have achieved a better classification performance. To this respect, it is worth noting that CMSE has been able to predict correctly a $20 \%$ of cases more than the remaining indices (see Table 3 ).

The estimated SampEn values in this study are slightly higher to those given in [13] (i.e., $0.074 \pm 0.015$ for NSR maintenance and $0.098 \pm 0.013$ for $\mathrm{AF}$ relapsing), although they exhibit a similar behavior. The difference between the results presented here and those in [13] could be associated with two aspects. On the one hand, the SampEn computation parameters, and, on the other hand, the MAW extraction algorithm. Whereas this work considered 30s length ECG segments and a value of 0.2 times the signal standard deviation for $r$, in [13] 10s length intervals were analyzed and a value of 0.25 was considered for $r$. Nonetheless, it should be noted that the results given in $[11,13]$ are also different, although they analyzed the same patients and used the same parameters for SampEn computation.

Moreover, bearing in mind that the AA signal often presents low-frequency residue, the MAW extraction process plays a key role for its accurate complexity characterization. To this respect, while the MAW was estimated by a band-pass FIR filtering in [11], an algorithm based on wavelet decomposition was used in [13], and a Type II Chebychev IIR filter has been considered in the present work. The results in [13] provided a higher specificity than the ones obtained in [11] $(79 \%$ vs. $59.1 \%$ ) and similar sensitivity (91\% vs. 90.2\%). These findings suggest that the better MAW estimation, the better results in predicting AF recurrence after ECV.

On the other hand, it is worth noting that no previous work has made use of multiscale entropy approaches for AA characterization, nor ECV outcome prediction. Nonetheless, Wavelet Entropy was considered in [14], this measure provides an spatio-temporal characterization of the AA. Wavelet entropy values were higher for AF relapsing $(0.627 \pm 0.021)$ than for NSR maintenance $(0.512 \pm 0.0025)$, obtaining a diagnostic accuracy of $84.75 \%$. These results are in line with those reported by CMSE at scale 19 (see Table 3), but their discriminant ability was lower. Anyway, these findings suggest that the most relevant nonlinear dynamics in the AA signal for ECV outcome prediction can be found at large time scales.

Summarizing, the use of nonlinear dynamics indexes, particularly CMSE, to characterize the AA signal extracted from the surface ECG enables a better ECV outcome prediction than other common parameters such as the DAF, FWAn and SampEn, providing a diagnostic accuracy higher than $90 \%$.

\section{ACKNOWLEDGMENT}

This work has been funded by projects: DPI2007-83952-C3 from MINECO/AEI/FEDER EU, SBPLY/17/180501000411 from "Junta de Castilla la Mancha" and AICO/2019/036 from "Generalitat Valenciana".

\section{REFERENCES}

[1] K. Miyazawa, G. Lip, “Atrial fibrillation”, Medicine, 46(10), 2018, pp. 627-631.

[2] J. Piccini, L. Fauchier, "Rhythm control in atrial fibrillation", The Lancet 2016, 388(10046), pp.829-840.

[3] C. Gutierrez, D. Blanchard, "Atrial fibrillation: diagnosis and treatment". Am Fam Physician, 83(1), 2011, pp.61-68.

[4] S. Jaakkola, G. Lip, F. Biancari, "Predicting unsuccessful electrical cardioversion for acute atrial fibrillation (from the AF-CVS score)", Am J Cardiol, 2017, 119(5), pp. 749-752.

[5] J. Andersson, M. Rosenqvist, P. Tornvall, K. Boman, "NTproBNP predicts maintenance of sinus rhythm after electrical cardioversion", Thromb Res, 135(2), 2015, pp.289-291

[6] R. Melduni, H. Lee, K. Bailey K, et al., "Real-time physiologic biomarker for prediction of atrial fibrillation recurrence, stroke, and mortality after electrical cardioversion: A prospective observational study", Am Heart J, 170(5), 2015, pp. 914-922

[7] R. Cervigón, J. Moreno,J. Millet, F. Castells, "Multiscale principal component analysis to predict atrial fibrillation reversion to sinus rhythm", Comp in Cardiol, 2016 Sep, pp. 465-468

[8] A. Seeck, W. Rademacher, C. Fischer, et al., "Prediction of atrial fibrillation recurrence after cardioversion-interaction analysis of cardiac autonomic regulation", Med Eng Phys, 35(3), 2013, pp. 376-382

[9] C. Luong, D. Thompson, K. Gin, et al., "Usefulness of the atrial emptying fraction to predict maintenance of sinus rhythm after direct current cardioversion for atrial fibrillation", Am J Cardiol 118(9), 2016, pp. 1345-1349

[10] R. Alcaraz, J. Rieta, "Time and frequency recurrence analisis of persistent atrial fibrillation after electrical cardioversion", Physiol Meas, 30, 2009, pp.479-489

[11] R. Alcaraz, J. Rieta, "A non-invasive method to predict electrical cardioversion outcome of persistent atrial fibrillation", Med Biol Eng Comp, 46(7), 2008, pp. 625-635

[12] R. Alcaraz, D. Abásolo, R. Hornero, J. "Optimal parameters study for sample entropy-based atrial fibrillation organization analysis", Comp Meth Prog Biomed, 99(1), 2010, pp. 124-132

[13] R. Alcaraz, F. Hornero, J. Rieta, "Noninvasive time and frequency predictors of long-standing atrial fibrillation early recurrence after electrical cardioversion: predictors of cardioversion outcome", Pacing Clin Electrophysiol, 34(10), 2011, pp. 1241-1250

[14] R. Alcaraz, J. Rieta, "Application of wavelet entropy to predict atrial fibrillation progression from the surface ECG”, Comp Math Meth Med, 2012, pp. 245213-9

[15] J. Richman, J. Moorman, "Physiological time-series analisis using approximate entropy and sample entropy", Am J Physiol Heart Circ Physiol, 278(6), 2000, pp.H2039-H2049

[16] S. Wu, C. Wu, S. Lin, C. Wang, K. Lee, "Time series analysis using composite multiscale entropy.", Entropy, 15(3), 2013, pp. 1069-1084

[17] M. Costa, A. Goldberger, C. Peng, "Multiscale entropy análisis of complex physiologic time series", Phys Rev Lett, 89, 2002, pp. 068102

[18] L. Sörnmo, P. Laguna, "ECG signal processing in bioelectrical signal processing in cardiac and neurological applications", 1st edition, Elsevier Academic Press, 2005

[19] M. Stridh, L. Sörnmo, "Spatiotemporal QRST cancellation techniques for analysis of atrial fibrillation”, IEEE Trans Biomed Eng, 48(1), 2001, pp.105-111

[20] J. Behar, A. Johnson, G. Clifford, J. Oster, "Comparison of single channel fetal ECG extraction methods", Ann Biomed Eng, 42(6), 2014, pp.1340-1353

[21] A. Martínez, R. Alcaraz, J. Rieta, "Ventricular activity morphological characterization: ectopic beats removal in long term atrial fibrillation recordings", Comput Methods Programs Biomed, 109, 2013, pp.283292 